\title{
Correlations between electronic order and structural distortions and their ultrafast dynamics in the single-layer manganite $\mathrm{Pr}_{0.5} \mathrm{Ca}_{1.5} \mathrm{MnO}_{4}$
}

\author{
M. Porer, ${ }^{1}$ L. Rettig, ${ }^{1,}$ E. M. Bothschafter, ${ }^{1}$ V. Esposito,,${ }^{1,}$ R. B. Versteeg $\odot,{ }^{2}$ P. H. M. van Loosdrecht, ${ }^{2}$ M. Savoini, ${ }^{1,3}$ \\ J. Rittmann, ${ }^{1}$ M. Kubli, ${ }^{3}$ G. Lantz, ${ }^{3}$ O. J. Schumann $\odot,{ }^{2,}$ A. A. Nugroho $\odot,{ }^{4}$ M. Braden, ${ }^{2}$ G. Ingold, ${ }^{1,5}$ S. L. Johnson $\odot,{ }^{3}$ \\ P. Beaud $\odot,{ }^{1,5}$ and U. Staub $\odot 1, \S$ \\ ${ }^{1}$ Swiss Light Source, Paul Scherrer Institute, 5232 Villigen PSI, Switzerland \\ ${ }^{2}$ Institute of Physics II, University of Cologne, 50937 Cologne, Germany \\ ${ }^{3}$ Institute for Quantum Electronics, ETH Zürich, 8093 Zürich, Switzerland \\ ${ }^{4}$ Faculty of Mathematics and Natural Science, Institut Teknologi Bandung, Indonesia \\ ${ }^{5}$ SwissFEL, Paul Scherrer Institute, 5232 Villigen PSI, Switzerland
}

(Received 5 December 2019; accepted 24 January 2020; published 18 February 2020)

\begin{abstract}
Time-resolved $\mathrm{x}$-ray diffraction experiments on the half-doped single-layered manganite $\operatorname{Pr}_{0.5} \mathrm{Ca}_{1.5} \mathrm{MnO}_{4}$ are used to monitor the ultrafast photoinduced dynamics of the structural distortion associated with the charge and orbital ordering $(\mathrm{CO} / \mathrm{OO})$. As in the nonlayered three-dimensional counterpart, the ordered phase melts in less than $100 \mathrm{fs}$ after 800-nm photoexcitation and subsequently partially recovers due to thermal equilibration of electronic and vibrational systems. Photoexciting $\operatorname{Pr}_{0.5} \mathrm{Ca}_{1.5} \mathrm{MnO}_{4}$ below the transition temperature of a second structural phase transition that occurs around $146 \mathrm{~K}$ (deep inside the $\mathrm{CO} / \mathrm{OO}$ phase) releases this structural transition, but progresses on a much slower timescale. This additional reduction of crystal symmetry, which we ascribe to a further tilt of the oxygen octahedra, can thus be considered to be only weakly coupled to $\mathrm{CO} / \mathrm{OO}$. Furthermore, static hard-x-ray and resonant soft-x-ray diffraction at the $\mathrm{Mn} L_{2,3}$ edges experiments identify correlations between structural distortions and electronic order in thermal equilibrium.
\end{abstract}

DOI: 10.1103/PhysRevB.101.075119

\section{INTRODUCTION}

Doped three-dimensional manganites have attracted considerable interest [1] as they exhibit metal-insulator transitions and colossal magnetoresistance effects. Strong coupling of lattice, spin, charge, and orbital degrees of freedom in mixed valence manganites leads to complex phase diagrams. In particular, the Jahn-Teller distortion (JTD) couples electronic charge and orbital order $(\mathrm{CO} / \mathrm{OO})$ with a symmetry breaking of the crystal lattice [1]. Information on the couplings can be obtained by studying the temperature dependence of the individual degrees of freedom. In manganites, structural distortions go hand in hand with electronic ordering phenomena, and which degree of freedom drives the $\mathrm{CO} / \mathrm{OO}$ phase transition remains unknown. Resonant soft-x-ray diffraction of superlattice reflections combines spectroscopic information on the $\mathrm{OO}$ and JTD and is therefore a powerful tool to address these couplings [2]. In particular, a detailed understanding of the electronic ordering has been obtained from the study of superlattice reflections at the $\operatorname{Mn} L_{2,3}$ and the $\mathrm{O} K$ edges [3-7]. Theoretical calculations predicted a different spectral

\footnotetext{
* Present address: Fritz Haber Institute of the Max Planck Society, Faradayweg 4-6, 14195 Berlin, Germany.

${ }^{\dagger}$ Present address: Stanford Institute for Materials and Energy Sciences (SIMES), SLAC National Laboratory, Menlo Park, CA 94025, USA.

${ }^{\ddagger}$ Present address: Fraunhofer-Institut für NaturwissenschaftlichTechnische Trendanalyse, Euskirchen, Germany.

§urs.staub@psi.ch
}

behavior of reflections sensitive to the $\mathrm{OO}$ and those sensitive to the JTD even before the first experiments were performed [8]. Experimentally, different temperature dependencies at various spectral features give information on the coupling, which is connected to the driving force of the transition. In particular, investigations of the orbital $(1 / 41 / 40)_{c}$ reflection ( $c$ stands for cubic notation) in layered manganite $\mathrm{La}_{0.5} \mathrm{Sr}_{1.5} \mathrm{MnO}_{4}$ have been interpreted in terms of $\mathrm{OO}$ being the main order parameter of the phase transition [9]. Similar investigations have subsequently been reported in another manganite system [10].

The coupling of these electronic degrees of freedom and the crystal structure has gained renewed interest as ultrafast probes allow us now to disentangle the dynamics of the individual degrees of freedom in the time domain [11]. The ultrafast dynamics of electronic and magnetic phase transitions in manganites have been extensively studied [12-19]. In particular, resolving the structural dynamics in three-dimensional (3D) manganites following the photoinduced melting of the charge and orbital ordered $(\mathrm{CO} / \mathrm{OO})$ phase has been addressed in detail [11,20-24]. It has been shown that a single order parameter that depends only on the electronic excitation is sufficient to describe the photoinduced ultrafast phase transition of the entire system, including charge and structural degrees of freedom [11]. The excitation suppresses the electronic order instantly $[11,25]$ and the response of both the underlying antiferromagnetic order [14] and the JTD [11] occurs on the same timescale of about $100 \mathrm{fs}$. The atomic rearrangement of the heavy ions within the unit cell is completed within $1 \mathrm{ps,}$ and the lattice constants readjust within 10-20 ps [23]. 


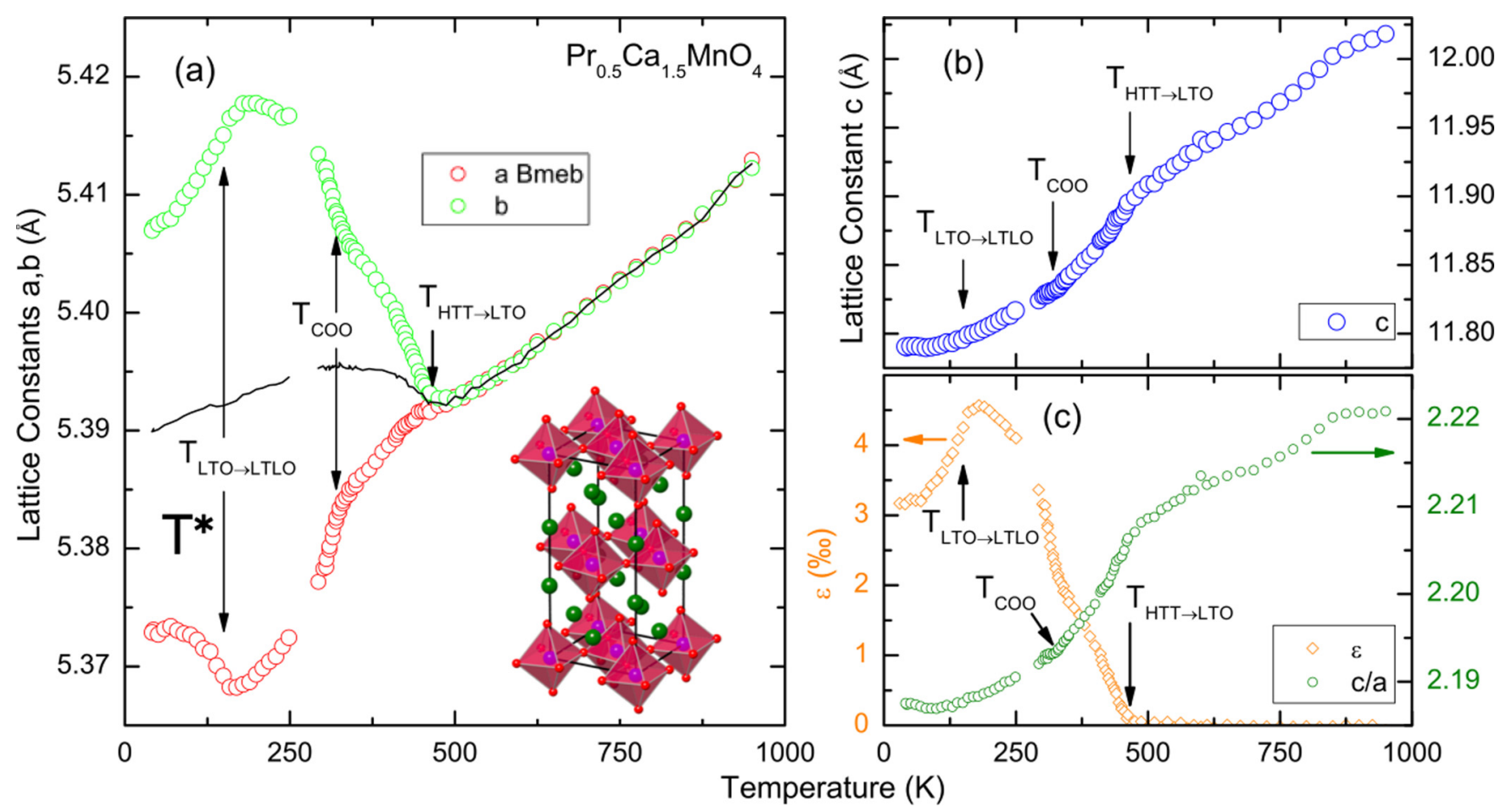

FIG. 1. Temperature dependence of the orthorhombic lattice parameters in PCMO4 determined by conventional powder x-ray diffraction. The splitting of the two in-plane parameters, shown in panel (a), sets in at the HTT-LTO transition at $466 \mathrm{~K}$ and becomes enhanced at the charge and orbital ordering transition at $320 \mathrm{~K}$; the orthorhombic splitting is reduced at the LTO-LTLO transition at $T^{*} \sim 146 \mathrm{~K}$ (see the text for more information). The black line denotes the average of the in-plane lattice parameters. The inset shows the $B m e b$ unit cell for $T>330 \mathrm{~K}$ (green spheres: $\mathrm{Pr} / \mathrm{Ca}$ ). The HTT-LTO transition is also observed as a kink in the $c$ parameter, shown in panel (b), and the charge and orbital ordering causes a change in the temperature slope. In contrast, there is no anomaly at $T^{*}$. Panel (c) shows the orthorhombic splitting and the $c / a$ ratio, which also exhibits anomalies at the respective transition temperatures.

Most investigations on electronic phase transitions in manganites concentrated on the three-dimensional distorted perovskites $R_{1-x} T_{x} \mathrm{MnO}_{3}$, with $R$ being a trivalent light lanthanide (La, or Pr ion) and $T$ a divalent metal ion such as $\mathrm{Ca}$ or Sr. However, much less is known on the structural dynamics of the layered counterparts, which have basically the same $\mathrm{CO} / \mathrm{OO}$ within the $\mathrm{MnO}$ layer [26]. However, the ordering perpendicular to the planes, which have a much larger separation due to the horizontal displacement of the oxygen octahedra (see, e.g., the inset of Fig. 1), exhibits significantly weaker correlations. Though the photoinduced $\mathrm{CO} / \mathrm{OO}$ dynamics and the dynamics of the antiferromagnetic ordering have been investigated for the single-layer $\mathrm{La}_{0.5} \mathrm{Sr}_{1.5} \mathrm{MnO}_{4}$ (LSMO4) system $[15,24]$, nothing is known on the ultrafast behavior of single-layer manganite $\mathrm{Pr}_{0.5} \mathrm{Ca}_{1.5} \mathrm{MnO}_{4}$ (PCMO4). The $\mathrm{CO} / \mathrm{OO}$ transition for PCMO4 occurs at $T \approx 320 \mathrm{~K}$. Associated with the metal-insulator-type transition, the hightemperature crystal structure (space group Bmeb) transforms to a structure that has an in-plane doubled unit cell with likely Pnma symmetry below $T_{\mathrm{CO} / \mathrm{OO}}[27,28]$. At $127 \mathrm{~K}$, there is an additional magnetic phase transition where the Mn spins order antiferromagnetically [29]. The lattice constants remain approximately constant between 130 and $200 \mathrm{~K}$ with $a=$ $5.37 \AA, b=5.42 \AA$, and $c=11.8 \AA$ (Bmeb symmetry) [28], which will be used further as a reference. An additional phase transition that is presumably structural in origin has been observed at $T^{*}=146 \mathrm{~K}$, below which additional reflections forbidden in Bmeb symmetry appear in x-ray and neutron diffraction experiments, such as, e.g., the (llll 10 ) reflection, indicating a further lowering in crystal symmetry [27].

In our present study, we clarify how the reduced dimensionality of the layered $\mathrm{Pr}_{0.5} \mathrm{Ca}_{1.5} \mathrm{MnO}_{4}$ (PCMO4) [28,29] influences the melting and recovery dynamics of the structural distortion associated with the CO/OO phase. In addition, we investigate the correlation between the $T^{*}$ transition and the $\mathrm{CO} / \mathrm{OO}$ order. To this end, we compare the ultrafast decay dynamics of a reflection sensitive to the $T^{*}$ transition with the structural dynamics associated with the suppression of the $\mathrm{CO} / \mathrm{OO}$ phase. For a qualitative understanding of the equilibrium coupling between electronic order and structural distortions, the temperature dependence of the $(01 / 20)$ superlattice reflection intensity was collected through static resonant soft$\mathrm{x}$-ray diffraction experiments. These results are compared to nonresonant hard-x-ray diffraction (HXRD) experiments on various superlattice reflections.

\section{EXPERIMENTS}

Single crystals of PCMO4 were grown using the floating zone technique as described in Refs. [27,30]. A half-moonshaped disk was cut out of the rod. The sample quality was verified with standard $\mathrm{x}$-ray diffraction characterization techniques and SQUID magnetometry. Temperature-dependent measurements of the lattice constants were performed on a D5000 x-ray powder diffractometer equipped with a liquidhelium cryostat and an Anton Paar furnace using a sample 
obtained by crushing a part of the single crystal. For the time-resolved experiments, a (2 70 ) surface was polished. The time-resolved $\mathrm{x}$-ray experiments were carried out at the FEMTO slicing source at the Swiss Light Source [31]. This source produced hard-x-ray pulses of $\sim 100 \mathrm{fs}$ duration. Using a double $\mathrm{Mo} / \mathrm{B}_{4} \mathrm{C}$ multilayer monochromator, an $\mathrm{x}$-ray energy of $7.1 \mathrm{keV}$ was selected, yielding a good compromise between the optimal source flux and minimum air-transmission losses. The penetration depth of the pump and the probe was approximately matched by using a small $0.5^{\circ}$ grazing incident angle of the x-ray beam. The estimated pump and probe depths are approximately 40 and $50 \mathrm{~nm}$ [32], respectively. The footprint of the x-ray probe beam on the sample surface was minimized by reducing the vertical beam size to $10 \mu \mathrm{m}$ [33]. The beam remained unfocused horizontally with a width of approximately $300 \mu \mathrm{m}$. The sample was excited with weakly focused $\left(520 \times 620 \mu \mathrm{m}^{2}\right) p$-polarized 100 -fs pulses at $800-\mathrm{nm}$ wavelength entering the sample at $10^{\circ}$ grazing incidence. The angle mismatch between the pump and the probe increases the time resolution to approximately $200 \mathrm{fs}$. The repetition rates of the optical pump and the $\mathrm{x}$-ray probe were 1 and $2 \mathrm{kHz}$, respectively. The probed diffracted intensity thus alternates between pumped and unpumped states of the sample, allowing us to compensate for slow drifts in the $\mathrm{x}$-ray source, pump laser, detector, and electronics [34]. The diffracted intensity of superlattice (SL) peaks was measured with an avalanche photodiode (APD), providing enhanced sensitivity for weak diffraction signals. The $\mathrm{x}$-ray energy is above the Mn $K$-edge and therefore x-ray fluorescence contributes significantly to a homogeneous background, which has been estimated from the constant baseline of rocking curve scans and was corrected for in the analysis. The crystal was cooled with a $\mathrm{N}_{2}$ cryoblower and the temperature has been set to $T \sim 100 \mathrm{~K}$. Static x-ray diffraction experiments were performed at the surface diffractometer of the X04SA Material Science beamline of the Swiss Light Source with an $\mathrm{x}$-ray energy of $10.8 \mathrm{keV}$ [35]. The sample was either cooled with a $\mathrm{N}_{2}$ cryoblower or glued on the cold finger of a $\mathrm{He}$ flow cryostat reaching 100 and $15 \mathrm{~K}$, respectively. Data were collected with a Pilatus pixel detector [36]. The resonant soft$\mathrm{x}$-ray diffraction experiments were performed at the RESOXS endstation [37] of the SIM beamline [38] in the vicinity of the $\operatorname{Mn} L_{2,3}$ edges. The sample was mounted with the [110] and [001] directions in the scattering plane. A continuous-flow helium cryostat was used to achieve sample temperatures between 50 and $350 \mathrm{~K}$. Experiments were performed with $\sigma$ and $\pi$ incident polarizations, corresponding to an incoming polarization perpendicular and parallel to the scattering plane. The outgoing polarization was not analyzed.

\section{RESULTS AND DISCUSSION}

\section{A. Structural transitions of PCMO4}

The temperature dependence of the lattice parameters that has been extracted from x-ray powder diffraction over a large temperature interval illustrates the various structural phase transitions occurring in PCMO4; see Fig. 1. The hightemperature tetragonal (HTT, space group $14 / \mathrm{mmm}$ ) phase transforms into the low-temperature orthorhombic phase
(LTO, space group Bmeb) at $466 \mathrm{~K}$ due to the tilting of the $\mathrm{MnO}_{6}$ octahedra, which commonly occurs in layered perovskites. In addition to the onset of orthorhombic splitting, there is a kink in the $c$ lattice parameter and an even more pronounced one in the $c / a$ ratio, because the average in-plane lattice constant expands in the LTO phase. The onset of charge and orbital ordering at $T_{\mathrm{CO} / \mathrm{OO}}$ enhances the orthorhombic splitting, but the impact on the average in-plane constant and on $c$ is opposite to that at the onset of the tilting. At the lower structural transition $T^{*}$ the orthorhombic splitting is reduced, but this effect is not associated with the onset of AFM ordering that occurs at even lower temperature. Below $T^{*}$ in neutron diffraction, an additional reflection emerges, (110) in orthorhombic notation [26,27,39], that signals further symmetry reduction. In accordance with the observation in various cuprates [40] as well as in nickelate and cobaltate compounds [41] of the same layered perovskite structure, we attribute this low-temperature structural phase transition to the change of the tilting scheme from LTO to the low-temperature less orthorhombic (LTLO, space group Pccn) one. In space group Bmeb, the $(h k 0)$ with $h, k=$ odd reflections are forbidden by the $b$ glide mirror symmetry, but these reflections are allowed in Pccn [and also in the low-temperature tetragonal (LTT, space group $P 4_{2} / \mathrm{ncm}$ ) phase]. The change in the tilting scheme from LTO to LTLO is a rather common phenomenon, and the fact that it occurs clearly above the onset of AFM order disagrees with the previously given interpretation of strong magnetoelastic coupling [29].

Doubling of the Bmeb unit cell along the $b$ axis induced by the $\mathrm{CO} / \mathrm{OO}$ yields a nonzero structure factor of reflections of type $(h k / 2 l)$ in orthorhombic notation [26,39]. Figure 2 shows the temperature dependence of the $(23 / 20)$ reflection (blue curve), which samples the in-plane structural distortion

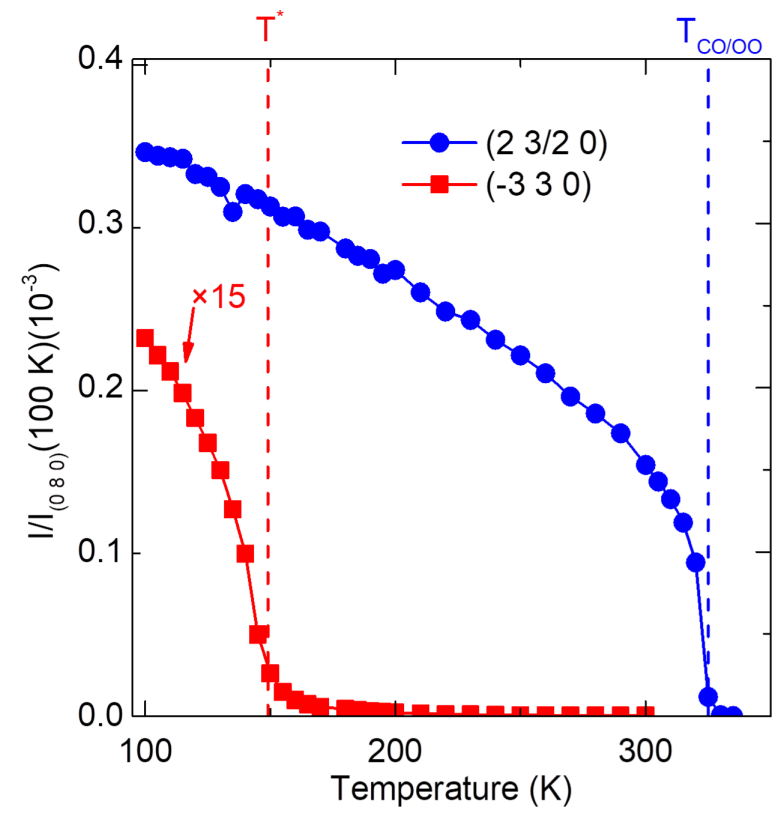

FIG. 2. Integrated intensity of the $\left(\begin{array}{lll}2 & 3 / 2 & 0\end{array}\right)$ and $\left(\begin{array}{lll}-3 & 3 & 0\end{array}\right)$ superlattice reflections (Bmeb notation) as a function of temperature normalized to the intensity of the $\left(\begin{array}{lll}0 & 8 & 0\end{array}\right)$ main structural reflection taken at $100 \mathrm{~K}$. 


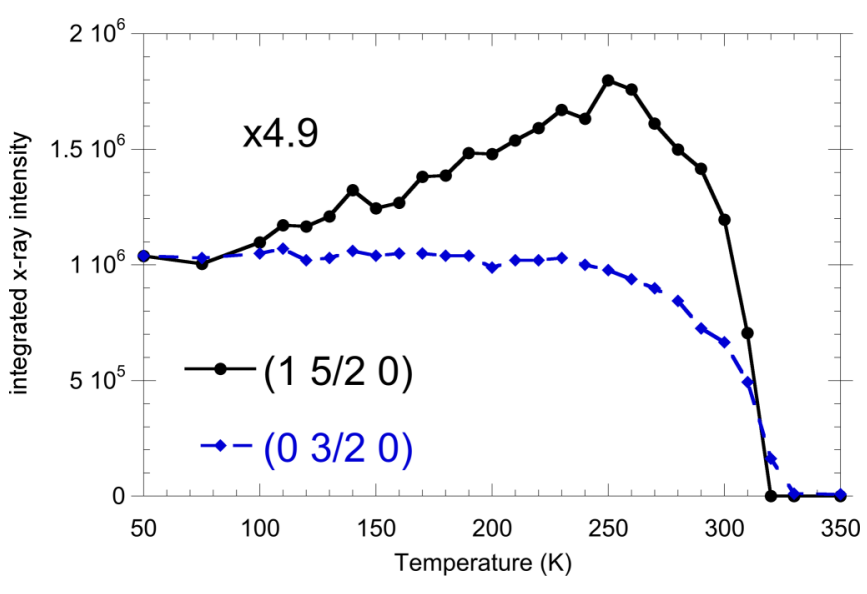

FIG. 3. Temperature dependence of integrated x-ray intensities of selected superlattice reflections.

and is insensitive to any structural distortions perpendicular to the plane as its Miller index $l$ is zero. Its intensity $I$ with changing temperature reflects the square of the order parameter of the $\mathrm{CO} / \mathrm{OO}$ phase, and it is not visibly affected by the LTO-LTLO transition at $T^{*}$. This reflection has been chosen for a better comparison to a previous pump-probe study on the 3D manganite LPCMO [42], which has a similar inplane $\mathrm{CO} / \mathrm{OO}$ transition and correspondingly similar in-plane distortions. The temperature dependence of the nonresonant $\mathrm{x}$-ray intensity of the $(-330)$ reflection (forbidden in Bmeb symmetry) is shown in Fig. 2 as red data points. The intensity of the $(-330)$ reflection is more than an order of magnitude smaller than that of the $(23 / 20)$ reflection, in agreement with the weakness of these reflections observed in other layered perovskites exhibiting the same LTO-LTLO transition [40,41].

Further in-plane reflections are shown in Fig. 3, and they exhibit a different temperature dependence than the $(23 / 20)$ reflection. The in-plane reflection $(03 / 20)$, which is only sensitive to the doubling of the unit cell, saturates around $250 \mathrm{~K}$ and the $(15 / 20)$ reflection shows a maximum at approximately $250 \mathrm{~K}$. This reflection is also significantly weaker than the $(23 / 20)$ reflection (see Fig. 2). However, both reflections probe structural distortions induced by the in-plane $\mathrm{CO} / \mathrm{OO}$. These strongly different temperature dependences show that there are other crystallographic modes than the JTD mode for properly describing the crystal structure below the $\mathrm{CO} / \mathrm{OO}$ phase transition as well as for the structural transition $T^{*}$.

\section{B. Electronic order}

To assess the relation of the unexplored structural transition at $T^{*}=146 \mathrm{~K}$ [27] to the CO/OO phase, we will first look directly at the temperature dependence of the orbital order by resonant soft-X-ray diffraction. The $\mathrm{OO}$ can be directly accessed at the $\operatorname{Mn} L_{2,3}$ edges by studying the energy and temperature dependence of the $(01 / 20)$ reflection, which has been done in detail in 3D crystals [5,43] and thin films of PCMO [44,45] as well as in the single-layer LSMO4 $[3,4,6,9]$. The spectral shape of the $(01 / 20)$ reflection in PCMO4 is shown in Fig. 4 and is very similar compared to that found for

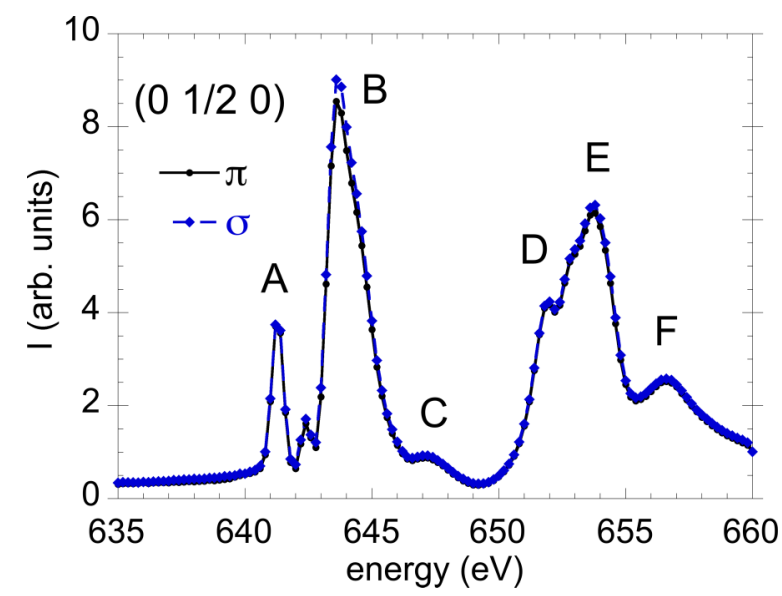

FIG. 4. Energy dependence of the OO $\left(0 \frac{1}{2} 20\right)$ peak intensity taken at $T=50 \mathrm{~K}$ for incident $\pi$ and $\sigma$ polarization. Different spectral features are labeled as in Ref. [9].

single-layer LSMO4. Therefore, we label the spectral features according to Ref. [9].

The temperature dependence of this reflection taken at different spectral features (energies) is shown in Fig. 5. As for LSMO4 [9], the shape of the temperature dependence depends strongly on the $\mathrm{x}$-ray energy with a similar behavior for equivalent spectral features [9]. The intensity of the highest energy feature $F$ does not have a regular order-parameter behavior; it peaks around $170 \mathrm{~K}$ for PCMO4. In LSMO4 this feature has a smooth temperature dependence and is only weakly temperature-dependent below $150 \mathrm{~K}$ with a broad maximum around $125 \mathrm{~K}$. The feature with the largest intensity at the $L_{3}$ edge $(B)$ shows in both cases a more regular order-parameter

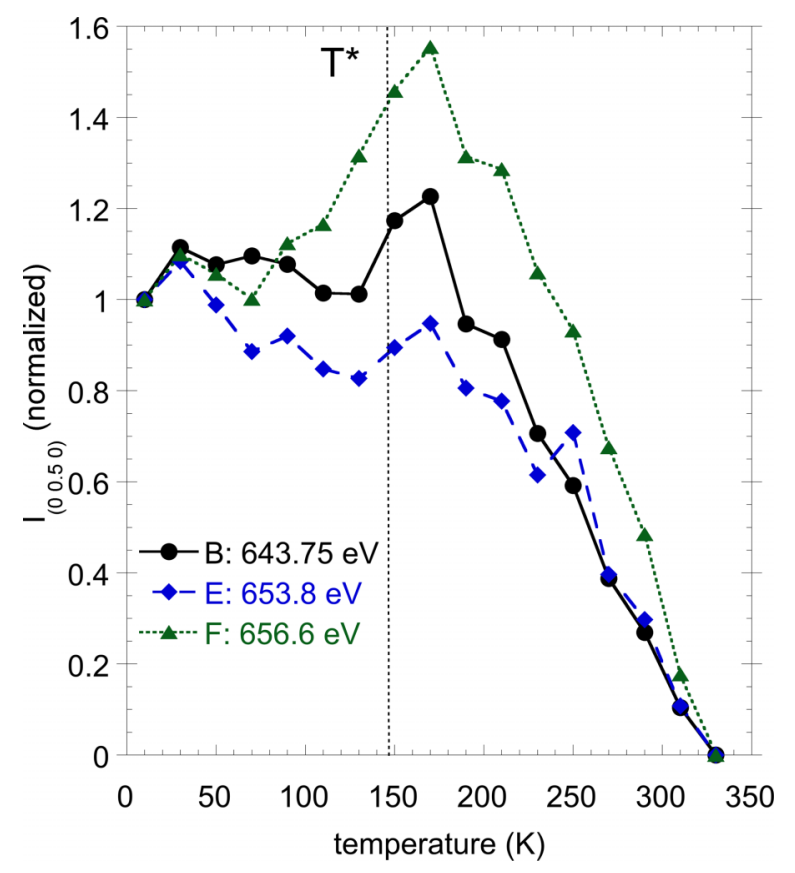

FIG. 5. Temperature dependence of the $\left(0 \frac{1}{2} 20\right)$ integrated reflection intensity taken at the spectral features $B, E$, and $F$ as marked in Fig. 4. The dotted vertical line represents the temperature of $T^{*}$. 


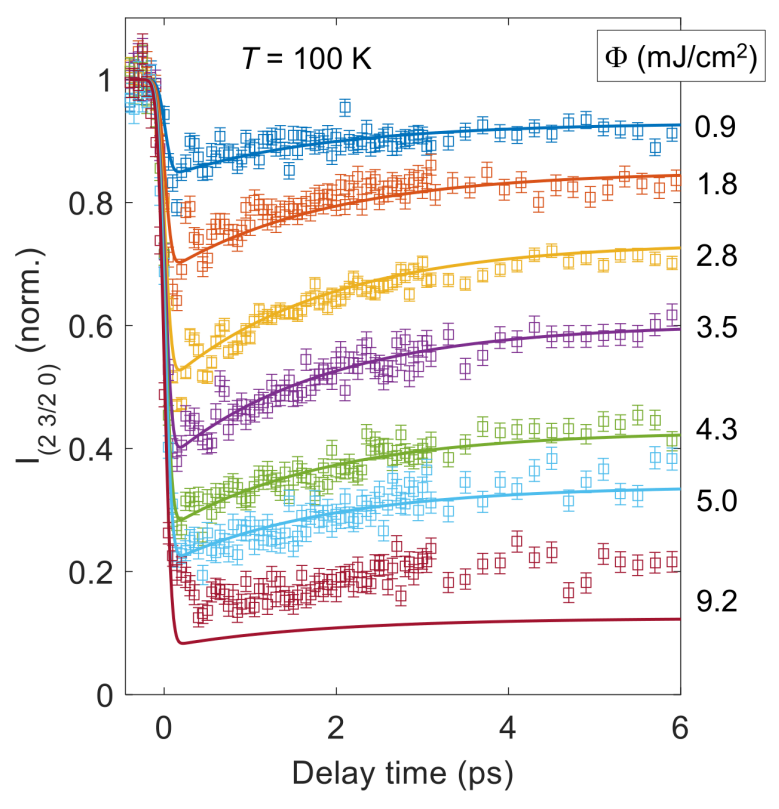

FIG. 6. Dynamics of the normalized (23/20) reflection intensity (data points) of bulk PCMO4 upon photoexcitation with 100-fs pulses centered at $800 \mathrm{~nm}$ for various absorbed pump fluences $\Phi$. The error bars indicate photon counting statistics. Solid lines result from numerical adaption of the model described in the main text.

behavior, i.e., it increases continuously for decreasing temperatures. The deviation from a regular order-parameter behavior is more pronounced for PCMO4 compared to LSMO4. These deviations are expected to be related to the differences in crystal structure of the two single-layer compounds. Whereas the crystal symmetry remains "quasi" tetragonal for LSMO4, the ground state of PCMO4 is clearly orthorhombic. In addition, PCMO4 shows an additional structural phase transition at $T^{*}$. The transition temperature $T^{*}$ lies lower in temperature than the maximum in the temperature dependence of the electronic spectral feature $F$, which makes a relation to $T^{*}$ questionable. However, the turnover in the temperature dependence of the spectral feature $F$ is likely related to structural modifications that are sensitive to the in-plane $b$ axis such as the JTD predicted in Ref. [9].

\section{Ultrafast dynamics of the $\mathrm{CO} / \mathrm{OO}$ induced structural distortion}

Figure 6 shows the normalized intensity of the (2 3/2 0) superlattice reflection as a function of the pump-probe delay $t$ for a series of excitation fluences $\Phi$ measured at $T=100 \mathrm{~K}$. Independent of $\Phi$, the optical excitation leads to a fast drop of the diffraction intensity on a timescale comparable to the experimental time resolution. Subsequently, the diffracted intensity partially recovers. Furthermore, we observe weak oscillatory dynamics superimposed on the recovery, which we resolve with better statistics for a selected excitation fluence (Fig. 7).

The initial excitation process most likely excites an electron within the Jahn-Teller-split Mn $3 d e_{g}$ states or between $\mathrm{Mn}^{3+}$ and $\mathrm{Mn}^{4+}$ similarly to the 3D system [20]. Both processes directly counteract the Jahn-Teller effect and lead to a

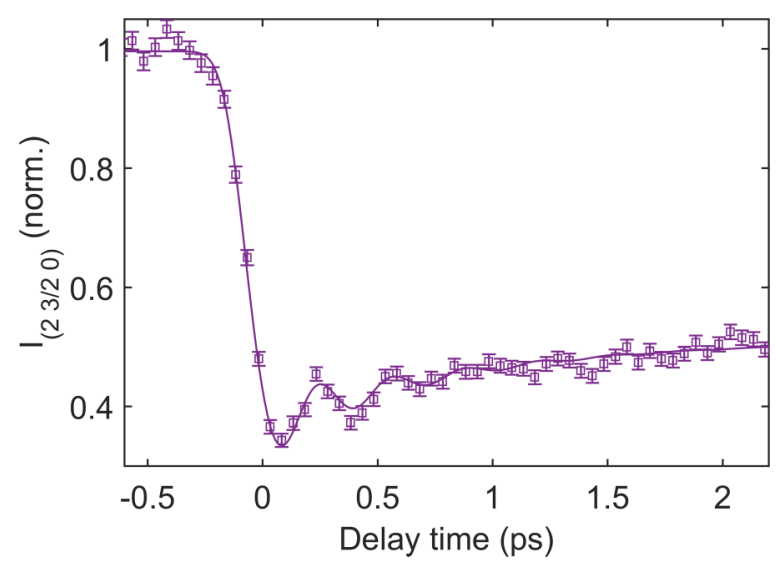

FIG. 7. Dynamics of the normalized (2 3/2 0) reflection intensity measured with improved statistics for a pump fluence $\Phi$ of $3.5 \mathrm{~mJ} / \mathrm{cm}^{2}$ at $T=100 \mathrm{~K}$. The line represents the fit as explained in the text.

suppression of $\mathrm{CO} / \mathrm{OO}$ and the structural JTD. Independent of the origin of the direct excitation process, the electronic system thermalizes faster than our experimental time resolution and randomizes the $\mathrm{Mn}^{3+}$ and $\mathrm{Mn}^{4+}$ states that destroy the $\mathrm{CO} / \mathrm{OO}$ state.

To describe the structural dynamics, we employ the single time-dependent order-parameter model that has been developed to describe the ultrafast phase transition of the 3D system [11]. Here we use the amplitude of the structural JTD as a direct measure for the order parameter of the transition. Following the approach of Refs. [11] and [46], we phenomenologically describe the normalized order parameter $\eta$ as a function of the deposited energy per volume $n$ and the critical energy density $n_{c}$ :

$$
\eta=\left(1-\frac{\min \left(n, n_{c}\right)}{n_{c}}\right)^{\gamma} .
$$

If $n>n_{c}$, the phase transition to the $\mathrm{CO} / \mathrm{OO}$ disordered state occurs and the order parameter vanishes. The timedependent model [11] accounts for a partial recovery of the order parameter, which is enabled by the energy dissipation from the electronic to the phonon system for deposited energy densities $n$ below $n_{c}$. To account for the process of energy deposition by the laser pulse and the subsequent dissipation of energy from the electronic to the lattice system, the energy density in the electronic system is described as being timedependent $[11,46]$ :

$$
n(t)=\left[n_{0}(t)-\alpha n_{c}\right] e^{-\frac{t}{\tau}}+\alpha n_{c} \text { for } t \geqslant 0
$$

with

$$
\alpha=1-\left(1-\frac{n_{0}(t)}{n_{c}}\right)^{\frac{\gamma}{\gamma_{0}}}
$$

and

$$
n_{0}(t)=\frac{1}{2} n_{0}\left[1+\operatorname{erf}\left(\frac{t}{\tau_{L}}\right)\right]
$$


Here, $\tau$ is an effective relaxation time describing the equilibration of the electron and phonon system, and $n_{0}$ is the total energy deposited by the pump laser pulse of duration $\tau_{\mathrm{L}}$. Furthermore, $\gamma_{0}$ and $\gamma$ are the critical exponents with respect to the initial excitation $(t \sim 0)$ and after equilibration $(t \gg \tau)$.

Here we do not imply a linear dependence between the reduction of the scattering intensity $I$ and $n_{0}$ for $t \sim 0$ (note that $\left.I \propto \eta^{2}\right)$. This is achieved by leaving $\gamma_{0}$ as a free parameter of the model $\left(\gamma_{0}=1 / 2\right.$ in Refs. $\left.[11,46]\right)$.

We follow the approach of Ref. [11] and model the transient reflection intensity considering a depth-dependent excitation density using an estimated penetration depth of 51 and $40 \mathrm{~nm}$ for the x-ray probe and the laser excitation, respectively. The total depth considered by the present model is $400 \mathrm{~nm}$ and consists of 400 layers. This allows us to fit the dynamics for all excitation fluences shown in Fig. 6 simultaneously (Fig. 6, solid lines). We account for the experimental time resolution of $\sim 200$ fs by convolution of $\eta^{2}(t)$ with a Gaussian function. We do not include the coherent oscillations of the Jahn-Teller soft mode by assuming that the JTD adiabatically follows the transient minimum position of the atomic potential (i.e., the JT mode is considered massless).

Fitting of the model yields $n_{c}=9 \times 10^{2} \mathrm{~J} / \mathrm{cm}^{3}$, which is about twice as high as in the 3D system [11]. This is in line with the higher ordering temperature of PCMO4, which suggests that more energy is needed to melt the ordered phase. The subsequent recovery occurs on a timescale of $\tau=1.85 \pm$ $0.5 \mathrm{ps}$, which is slightly slower than in the $3 \mathrm{D}$ system. This could indicate a smaller $e$-ph coupling of the layered system. Furthermore, we extract a critical exponent of the thermalized system of $\gamma=0.29 \pm 0.03$ and for the initial excitation of $\gamma_{0}=0.69 \pm 0.05$. While $\gamma$ is comparable to the 3D system, a higher value of $\gamma_{0}$ is required to obtain a good fit to the experimental data for PCMO4.

The fast-initial drop after photoexcitation occurs within the time resolution of the experiment. As in the 3D system, the fast dynamics indicates that the structural distortion is promptly released by the suppression of $\mathrm{CO} / \mathrm{OO}$. The critical behavior of the squared order parameter against the excitation fluence is shown in Fig. 8, which compares the experimental scattering intensity at early and late times. We find the scaling to be qualitatively similar to that for $800 \mathrm{~nm}$ excitation in 3D manganites [11] and nickelates [46]. The deviation of the model to the highest-fluence data is likely due to the remaining weakly excited regions of the probed volume due to an uncertainty in the determination of the pump and probe depths, particularly for bulk crystals. In addition, the high fluence data are further affected by an incomplete recovery between successive pump pulses, as was recently observed for 3D PCMO [23]: For fluences higher than required to fully melt the $\mathrm{CO} / \mathrm{OO}$ order at the surface, the diffraction intensity no longer completely recovers between successive pump pulses due to the first-order character of the $\mathrm{CO} / \mathrm{OO}$ transition, which results in slow dynamics of domain growth. The highest fluence time trace where this effect is largest was thus not included in the fitting procedure.

Figure 7 shows the trace for a pump fluence $\Phi$ of $3.5 \mathrm{~mJ} / \mathrm{cm}^{2}$ with improved statistics, giving clear evidence of a displacive lattice excitation. We employ a phenomenological fit function to extract the oscillation frequency:

$$
\begin{aligned}
\frac{I(t)}{I(0)}= & 1-\Theta\left(t-t_{0}\right)\left(A e^{-\left(t-t_{0}\right) / \tau_{1}}\right. \\
& \left.+B e^{-\left(t-t_{0}\right) / \tau_{2}} \cos \left[2 \pi v\left(t-t_{0}\right)\right]\right),
\end{aligned}
$$

where $\Theta$ is the Heaviside function, and $A$ and $B$ represent amplitudes of the recovery and the phonon mode, respectively. We find a frequency of the displacive mode $v=3.15 \mathrm{THz}$ that is approximately $30 \%$ higher than in $3 \mathrm{D} \mathrm{Pr}_{0.5} \mathrm{Ca}_{0.5} \mathrm{MnO}_{3}$ [11], where it has been interpreted to contain motions of the heavy Pr ions [20], which seems similar to PCMO4 [47]. The slightly higher frequency as compared to the $3 \mathrm{D}$ system might be explained by a larger Coulomb repulsion caused by shorter in-plane bond distances. Note that the average in-plane lattice constants are reduced and more anisotropic [48] compared to that of the 3D analogon. Similar to the 3D compound, we assign this coherent modulation to atomic motions along the transition coordinates, triggered by the partial melting of the $\mathrm{CO} / \mathrm{OO}$ and subsequent relaxation of the JTD.

\section{Ultrafast dynamics of structural distortion below $T^{*}$}

To further address a possible correlation between the $\mathrm{CO} / \mathrm{OO}$ and the structural second-order-like phase transition around $T^{*}$, we investigated the photoinduced ultrafast dynamics of the $(-330)$ reflection, which appears below $T^{*}$. The time trace of the reflection intensity after photoexcitation is shown in Fig. 9. As this reflection is much weaker, it required longer integration times to achieve reasonable statistics in the data set. This reflection shows a transient suppression that is significantly slower compared to the electronically driven relaxation of the distortion associated with the $\mathrm{CO} / \mathrm{OO}$, where the observed intensity drop was limited by the time resolution of the experiment. A fit using single exponential decay yields a time constant of 0.5 ps. This much slower structural relaxation dynamics is consistent with the absence

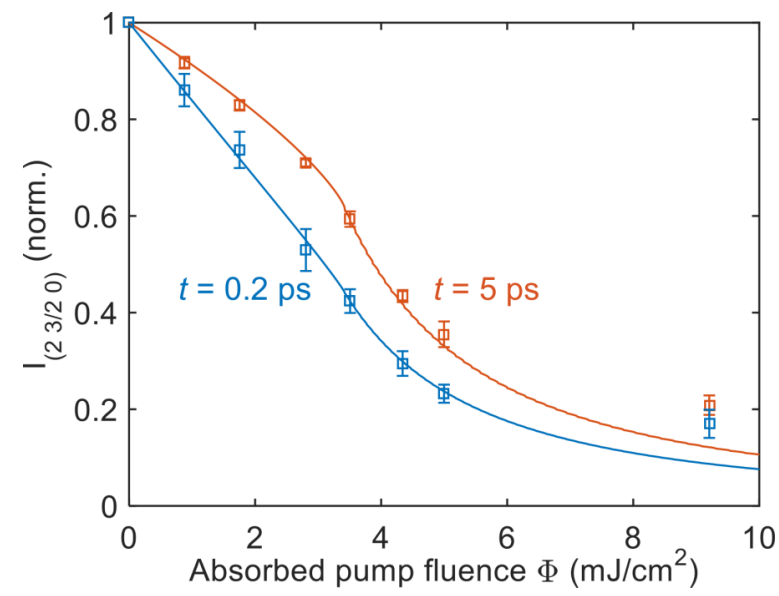

FIG. 8. Normalized x-ray scattering intensity of the (2 3/2 0$)$ reflection averaged in the vicinity of 0.2 and 5 ps time delay as a function of the pump fluence. Error bars indicate the standard deviation of data points within the averaging windows $(0.1-0.3$ and 4.5-5.5 ps, respectively). The lines are fits of the model to the data as explained in the text. 


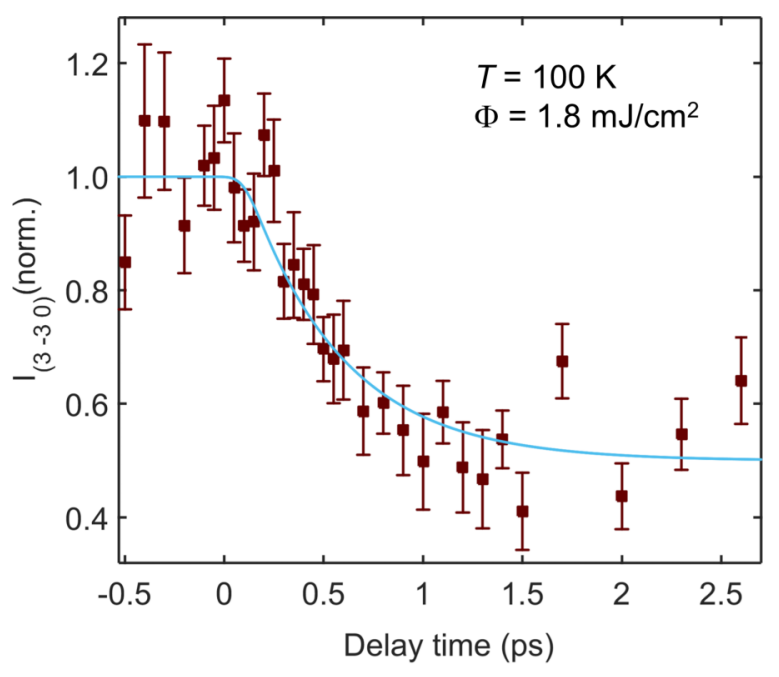

FIG. 9. Pump-induced dynamics of the $\left(\begin{array}{lll}-3 & 3 & 0\end{array}\right)$ superlattice peak. Solid curve: phenomenological fit.

of a significant coupling to the $\mathrm{CO} / \mathrm{OO}$, which agrees with the interpretation of the resonant soft-x-ray diffraction data. Assuming the transition at $T^{*}$ is displacive in nature, the slow relaxation indicates a strongly damped, very low energy soft mode associated with this phase transition. Such a behavior is in accordance with the Pccn LTLO symmetry that bridges the LTO and LTT phases and that thus exhibit a soft tilting mode. Interestingly, the observed timescale here is much slower than that observed for a phonon-driven phase transition in simple perovskites. Whereas for $\mathrm{SrTiO}_{3}$ [49] a much faster decrease of the corresponding superlattice reflection has been found, an ultrafast increase of the reflection has been found in $\mathrm{EuTiO}_{3}$, indicative of a transient increase in the structural order parameter [50]. In both cases, however, the dynamics is driven by changes of the structural soft-mode potential due to photodoping, most likely in contrast to the structural $T^{*}$ transition in PCMO4.

\section{CONCLUSION}

Ultrafast $\mathrm{x}$-ray diffraction was used to study the time dependence of superlattice reflections sensitive to the $\mathrm{CO} / \mathrm{OO}$ ground state of layered PCMO4. They show similar dynamics compared to three-dimensional PCMO after an ultrashort optical pulse excites the system. It indicates that the $\mathrm{CO} / \mathrm{OO}$ melting and recovery dynamics is tightly coupled to the JTD distortion also in layered manganites. We further argue that the additional structural phase transition, occurring deep inside the charge order phase (at $T^{*}$ ), can be attributed to the change of the tilting scheme from LTO to LTLO. This structural transition behaves differently when probing the respective symmetry-breaking reflections. These characteristic LTLO reflection intensities are suppressed on significantly slower timescales, indicative of a decoupling of this structural transition from the CO/OO. The static resonant soft-x-ray diffraction experiments on $\mathrm{OO}$ superlattice reflections find a temperature dependence of the reflection intensity, which depends on the energy of the $\mathrm{x}$ rays, but they do not show any indication of an anomaly at $T^{*}$. This supports the interpretation of insensitivity of the LTO-LTLO transition to the $\mathrm{CO} / \mathrm{OO}$. The variation in the temperature dependence of the reflection taken at various $\mathrm{x}$-ray energies at the absorption edge shows that other structural modes couple to the $\mathrm{OO}$ in addition to the JTD. This view is supported by the differences in the temperature dependence between structural reflections sensitive to different structural modifications. In summary, it shows that the layered manganites based on Pr have a much richer interplay of crystal structure and its electronic properties than three-dimensional half-doped manganites.

\section{ACKNOWLEDGMENTS}

We thank D. Meister and A. Oggenfuss for technical support at the X04SA and FEMTO beamlines, and D. Grolimund for supporting the time-resolved "femtoslicing" experiments at the X05SA beamline. We further acknowledge help by E. Pomjakushina and S. Stutz with preparation of the sample surface. The research leading to these results has received funding from the Swiss National Science Foundation and its National Center of Competence in Research, Molecular Ultrafast Science and Technology (NCCR MUST) and Materials' Revolution: Computational Design and Discovery of Novel Materials (NCCR MARVEL). L.R. acknowledges funding from the DFG in the Emmy Noether program under Grant No. RE 3977/1. E.M.B. acknowledges funding from the European Community's Seventh Framework Programme (FP7/2007-2013) under Grant Agreement No. 290605 (PSIFELLOW/COFUND). We acknowledge financial support of the Deutsche Forschungsgemeinschaft (DFG) through CRC 1238 (Project No. 277146847, subprojects A02, B04, and B05).
[1] M. B. Salamon and M. Jaime, Rev. Mod. Phys. 73, 583 (2001).

[2] J. Fink, E. Schierle, E. Weschke, and J. Geck, Rep. Prog. Phys. 76, 056502 (2013).

[3] S. B. Wilkins, P. D. Spencer, P. D. Hatton, S. P. Collins, M. D. Roper, D. Prabhakaran, and A. T. Boothroyd, Phys. Rev. Lett. 91, 167205 (2003).

[4] S. S. Dhesi, A. Mirone, C. D. Nadai, P. Ohresser, P. Bencok, N. B. Brookes, P. Reutler, A. Revcolevschi, A. Tagliaferri, O. Toulemonde, and G. v. d. Laan, Phys. Rev. Lett. 92, 056403 (2004).
[5] K. J. Thomas, J. P. Hill, S. Grenier, Y.-J. Kim, P. Abbamonte, L. Venema, A. Rusydi, Y. Tomioka, Y. Tokura, D. F. McMorrow, G. Sawatzky, and M. v. Veenendaal, Phys. Rev. Lett. 92, 237204 (2004).

[6] U. Staub, V. Scagnoli, A. M. Mulders, K. Katsumata, Z. Honda, H. Grimmer, M. Horisberger, and J. M. Tonnerre, Phys. Rev. B 71, 214421 (2005).

[7] S. Grenier, K. J. Thomas, J. P. Hill, U. Staub, Y. Bodenthin, M. Garcia-Fernandez, V. Scagnoli, V. Kiryukhin, S. W. Cheong, B. G. Kim, and J. M. Tonnerre, Phys. Rev. Lett. 99, 206403 (2007). 
[8] C. W. M. Castleton and M. Altarelli, Phys. Rev. B 62, 1033 (2000).

[9] U. Staub, V. Scagnoli, A. M. Mulders, M. Janousch, Z. Honda, and J. M. Tonnerre, Europhys. Lett. 76, 926 (2006).

[10] M. Garcia-Fernandez, U. Staub, Y. Bodenthin, V. Pomjakushin, A. Mirone, J. Fernandez-Rodriguez, V. Scagnoli, A. M. Mulders, S. M. Lawrence, and E. Pomjakushina, Phys. Rev. B 82, 235108 (2010).

[11] P. Beaud, A. Caviezel, S. O. Mariager, L. Rettig, G. Ingold, C. Dornes, S.-W. Huang, J. A. Johnson, M. Radovic, T. Huber, T. Kubacka, A. Ferrer, H. T. Lemke, M. Chollet, D. Zhu, J. M. Glownia, M. Sikorski, A. Robert, H. Wadati, M. Nakamur, M. Kawasaki, Y. Tokura, S. L. Johnson, and U. Staub, Nat. Mater. 13, 923 (2014).

[12] D. Polli, M. Rini, S. Wall, R. W. Schoenlein, Y. Tomioka, Y. Tokura, G. Cerullo, and A. Cavalleri, Nat. Mater. 6, 643 (2007).

[13] M. Rini, R. Tobey, N. Dean, J. Itani, Y. Tomioka, Y. Tokura, W. Schoenlein, and A. Cavalleri, Nature (London) 449, 72 (2007).

[14] M. C. Langner, S. Zhou, G. Coslovich, Y. D. Chuang, Y. Zhu, J. S. Robinson, W. F. Schlotter, J. J. Turner, M. P. Minitti, R. G. Moore, W. S. Lee, D. H. Lu, D. Doering, P. Denes, Y. Tomioka, Y. Tokura, R. A. Kaindl, and R. W. Schoenlein, Phys. Rev. B 92, 155148 (2015).

[15] H. Ehrke, R. I. Tobey, S. Wall, S. A. Cavill, M. Först, V. Khanna, T. Garl, N. Stojanovic, D. Prabhakaran, A. T. Boothroyd, N. Gensch, A. Mirone, P. Reutler, A. Revcolevschi, S. S. Dhesi, and A. Cavalieri, Phys. Rev. Lett. 106, 217401 (2011).

[16] H. Matsuzaki, H. Uemura, M. Matsubara, T. Kimura, Y. Tokura, and H. Okamoto, Phys. Rev. B 79, 235131 (2009).

[17] T. Mertelj, R. Yusupov, A. Gradisek, M. Filippi, W. Prellier, and D. Mihailovic, Europhys. Lett. 86, 57003 (2009).

[18] T. Q. Li, A. Patz, L. Mouchliadis, J. Q. Yan, T. A. Lograsso, I. E. Perakis, and J. G. Wang, Nature (London) 496, 69 (2013).

[19] L. Rettig, A. Caviezel, S. O. Mariager, G. Ingold, C. Dornes, S. W. Huang, J. A. Johnson, M. Radovic, T. Huber, T. Kubacka, A. Ferrer, H. T. Lemke, M. Chollet, D. Zhu, J. M. Glownia, M. Sikorski, A. Robert, M. Nakamura, M. Kawasaki, Y. Tokura, S. L. Johnson, P. Beaud, and U. Staub, Phys. Rev. B 99, 134302 (2019).

[20] P. Beaud, S. L. Johnson, E. Vorobeva, U. Staub, R. A. De Souza, C. J. Milne, Q. X. Jia, and G. Ingold, Phys. Rev. Lett. 103, 155702 (2009).

[21] A. Caviezel, U. Staub, S. L. Johnson, S. O. Mariager, E. Mohr-Vorobeva, G. Ingold, C. J. Milne, M. Garganourakis, V. Scagnoli, S. W. Huang, Q. X. Jia, S. W. Cheong, and P. Beaud, Phys. Rev. B 86, 174105 (2012).

[22] V. Esposito, M. Fechner, R. Mankowsky, H. Lemke, M. Chollet, J. M. Glownia, M. Nakamura, M. Kawasaki, Y. Tokura, U. Staub, P. Beaud, and M. Forst, Phys. Rev. Lett. 118, 247601 (2017).

[23] V. Esposito, L. Rettig, E. Abreu, E. M. Bothschafter, G. Ingold, M. Kawasaki, M. Kubli, G. Lantz, M. Nakamura, J. Rittman, M. Savoini, Y. Tokura, U. Staub, S. L. Johnson, and P. Beaud, Phys. Rev. B 97, 014312 (2018).

[24] M. Först, R. I. Tobey, S. Wall, H. Bromberger, V. Khanna, A. Cavalieri, Y.-D. Chuang, W.-S. Lee, R. Moore, W. F. Schlotter, J. J. Turner, O. Krupin, M. Trigo, H. Zheng, J. F. Mitchell, S. S. Dhesi, J. P. Hill, and A. Cavalleri, Phys. Rev. B 84, 241104(R) (2011).
[25] R. Singla, A. Simoncig, M. Först, D. Prabhakaran, A. L. Cavalieri, and A. Cavalleri, Phys. Rev. B 88, 075107 (2013).

[26] B. J. Sternlieb, J. P. Hill, U. C. Wildgruber, G. M. Luke, B. Nachumi, Y. Moritomo, and Y. Tokura, Phys. Rev. Lett. 76, 2169 (1996).

[27] O. J. Schumann, Ph.D. thesis, University of Cologne, 2010.

[28] I. P. Handayani, A. A. Nugroho, S. Riyadi, G. R. Blake, N. Mufti, T. T. M. Palstra, and P. H. M. van Loosdrecht, Phys. Rev. B 92, 205101 (2015).

[29] S. Chi, F. Ye, P. Dai, J. A. Fernandez-Baca, Q. Huang, J. W. Lynn, E. W. Plummer, R. Mathieu, Y. Kaneko, and Y. Tokura, Proc. Natl. Acad. Sci. (U.S.A.) 104, 10796 (2007).

[30] P. Reutler, O. Friedt, B. Buchner, M. Braden, and A. Revcolevschi, J. Cryst. Growth 249, 222 (2003).

[31] P. Beaud, S. L. Johnson, A. Streun, R. Abela, D. Abramsohn, D. Grolimund, F. Krasniqi, T. Schmidt, V. Schlott, and G. Ingold, Phys. Rev. Lett. 99, 174801 (2007).

[32] M. A. Majidi, E. Thoeng, P. K. Gogoi, F. Wendt, S. H. Wang, I. Santoso, T. C. Asmara, I. P. Handayani, P. H. M. van Loosdrecht, A. A. Nugroho, M. Rübhausen, and A. Rusydi, Phys. Rev. B 87, 235135 (2013).

[33] S. L. Johnson, P. Beaud, C. J. Milne, F. S. Krasniqi, E. S. Zijlstra, M. E. Garcia, M. Kaiser, D. Grolimund, R. Abela, and G. Ingold, Phys. Rev. Lett. 100, 155501 (2008).

[34] M. Saes, C. Bressler, F. van Mourik, W. Gawelda, M. Kaiser, M. Chergui, C. Bressler, D. Grolimund, R. Abela, T. E. Glover, P. A. Heimann, R. W. Schoenlein, S. L. Johnson, A. M. Lindenberg, and R. W. Falcone, Rev. Sci. Instrum. 75, 24 (2004).

[35] P. R. Willmott, D. Meister, S. J. Leake, M. Lange, A. Bergamaschi, M. Boge, M. Calvi, C. Cancellieri, N. Casati, A. Cervellino, Q. Chen, C. David, U. Flechsig, F. Gozzo, B. Henrich, S. Jaggi-Spielmann, B. Jakob, I. Kalichava, P. Karvinen, J. Krempasky, A. Ludeke, R. Luscher, S. Maag, C. Quitmann, M. L. Reinle-Schmitt, T. Schmidt, B. Schmitt, A. Streun, I. Vartiainen, M. Vitins, X. Wang, and R. Wullschleger, J. Synch. Rad. 20, 667 (2013).

[36] R. Dinapoli, A. Bergamaschi, B. Henrich, R. Horisberger, I. Johnson, A. Mozzanica, E. Schmid, B. Schmitt, A. Schreiber, X. Shi, and G. Theidel, Nucl. Instrum. Methods Phys. Res. Sect. A 650, 79 (2011).

[37] U. Staub, V. Scagnoli, Y. Bodenthin, M. García-Fernández, R. Wetter, A. M. Mulders, H. Grimmer, and M. Horisberger, J. Synch. Rad. 15, 469 (2008).

[38] U. Flechsig, F. Nolting, A. Fraile-RodrÍguez, J. Krempaský, C. Quitmann, T. Schmidt, S. Spielmann, and D. Zimoch, in SRI 2009, Tenth International Conference on Radiation Instrumentation, edited by R. Garrett, I. Gentle, K. Nugent, and S. Wilkins, AIP Conf. Proc. No. 1234 (AIP, New York, 2010), p. 319.

[39] D. Senff, O. Schumann, M. Benomar, M. Kriener, T. Lorenz, Y. Sidis, K. Habicht, P. Link, and M. Braden, Phys. Rev. B 77, 184413 (2008).

[40] J. M. Tranquada, G. D. Gu, M. Hucker, Q. Jie, H. J. Kang, R. Klingeler, Q. Li, N. Tristan, J. S. Wen, G. Y. Xu, Z. J. Xu, J. Zhou, and M. van Zimmermann, Phys. Rev. B 78, 174529 (2008).

[41] H. Ulbrich and M. Braden, Physica C 481, 31 (2012).

[42] A. Caviezel, S. O. Mariager, S. L. Johnson, E. Mohr-Vorobeva, S. W. Huang, G. Ingold, U. Staub, C. J. Milne, S. W. Cheong, and P. Beaud, Phys. Rev. B 87, 205104 (2013). 
[43] U. Staub, M. Garcia-Fernandez, Y. Bodenthin, V. Scagnoli, R. A. De Souza, M. Garganourakis, E. Pomjakushina, and K. Conder, Phys. Rev. B 79, 224419 (2009).

[44] H. Wadati, J. Geck, E. Schierle, R. Sutarto, F. He, D. G. Hawthorn, M. Nakamura, M. Kawasaki, Y. Tokura, and G. A. Sawatzky, New J. Phys. 16, 033006 (2014).

[45] M. Garganourakis, V. Scagnoli, S. W. Huang, U. Staub, H. Wadati, M. Nakamura, V. A. Guzenko, M. Kawasaki, and Y. Tokura, Phys. Rev. Lett. 109, 157203 (2012).

[46] V. Esposito, L. Rettig, E. M. Bothschafter, Y. P. Deng, C. Dornes, L. Huber, T. Huber, G. Ingold, Y. Inubushi, T. Katayama, T. Kawaguchi, H. Lemke, K. Ogawa, S. Owada, M. Radovic, M. Ramakrishnan, Z. Ristic, V. Scagnoli, Y. Tanaka, T. Togashi, K. Tono, I. Usov, Y. W. Windsor, M. Yabashi, S. L. Johnson, P. Beaud, and U. Staub, Struct. Dyn. 5, 064501 (2018).
[47] I. P. Handayani, Ph.D. thesis, University of Groningen, 2014.

[48] P. M. Woodward, T. Vogt, D. E. Cox, A. Arulraj, C. N. R. Rao, P. Karen, and A. K. Cheetham, Chem. Mater. 10, 3652 (1998).

[49] M. Porer, M. Fechner, E. M. Bothschafter, L. Rettig, M. Savoini, V. Esposito, J. Rittmann, M. Kubli, M. J. Neugebauer, E. Abreu, T. Kubacka, T. Huber, G. Lantz, S. Parchenko, S. Grubel, A. Paarmann, J. Noack, P. Beaud, G. Ingold, U. Aschauer, S. L. Johnson, and U. Staub, Phys. Rev. Lett. 121, 055701 (2018).

[50] M. Porer, M. Fechner, M. Kubli, M. J. Neugebauer, S. Parchenko, V. Esposito, A. Narayan, N. A. Spaldin, R. Huber, M. Radovic, E. M. Bothschafter, J. M. Glownia, T. Sato, S. Song, S. L. Johnson, and U. Staub, Phys. Rev. Res. 1, 012005 (2019). 